\title{
From a Boson to the Standard Model Higgs: A Case Study in Confirmation and Model Dynamics
}

\author{
Cristin Chall $^{*} \quad$ Martin King $^{\dagger} \quad$ Peter Mättig $^{\ddagger} \quad$ Michael Stöltzner $^{\S}$
}

April 19, 2019

\begin{abstract}
Our paper studies the anatomy of the discovery of the Higgs boson at the Large Hadron Collider (LHC). We investigate the phases of this discovery, which led to a crucial reconfiguration of the model landscape of elementary particle physics and eventually to a confirmation of the Standard Model (SM). A keyword search of preprints covering the electroweak symmetry breaking (EWSB) sector of particle physics, along with an examination of physicists' own understanding of the discovery as documented in semiannual conferences, has allowed us an empirical investigation of its model dynamics. From our analyses we draw two main philosophical lessons concerning the nature of scientific reasoning in a complex experimental and theoretical environment. For one, from a confirmation standpoint, some SM alternatives could be considered even more confirmed by the Higgs discovery than the SM. Nevertheless, the SM largely remains the commonly accepted account of EWSB. We present criteria for comparing degrees of confirmation and expose some limits of a purely logical approach to understanding the Higgs discovery as a victory for the SM. Second, we understand the persistence of SM alternatives in the face of disfavourable evidence by borrowing the Lakatosian concept of a research programme, where the core idea behind a group of models survives, while other aspects adapt to incoming data. In order to apply this framework to the model landscape of EWSB, we must introduce a new category of research programme, the modelgroup, and we test its viability using the example of composite Higgs models.
\end{abstract}

This is a pre-print of an article published in Synthese. The final authenticated version is available online at: https://doi.org/10.1007/s11229-019-02216-7.

\section{Introduction}

Our paper studies the anatomy of the recent discovery of the Higgs boson at the Large Hadron Collider (LHC) in Geneva. We investigate the various phases of this discovery, which led to a crucial reconfiguration of the model landscape of elementary particle physics and eventually to a confirmation of the Standard Model (SM). The philosophical lessons of our analysis concern the

\footnotetext{
* To contact, please write to Physikalisches Institut, Rheinische Friedrich-Wilhelms-Universität Bonn, Nussallee 12, 53115 Bonn, Germany; email: chall@physik.uni-bonn.de.

$\dagger$ To contact, please write to Physikalisches Institut, Rheinische Friedrich-Wilhelms-Universität Bonn, Nussallee 12, 53115 Bonn, Germany; email: king@physik.uni-bonn.de.

$\ddagger$ To contact, please write to Physikalisches Institut, Rheinische Friedrich-Wilhelms-Universität Bonn, Nussallee 12, 53115 Bonn, Germany; email: peter.mattig@cern.ch.

$\S$ To contact, please write to Department of Philosophy, University of South Carolina, 901 Sumter St., Columbia, SC 29208, United States of America; email: stoeltzn@mailbox.sc.edu.
} 
nature of confirmation in a complex experimental and theoretical environment and the dynamics of research programmes in a variegated landscape of models.

There were several physical reasons for doubting that the Higgs search at the LHC, would bring an immediate and straightforward confirmation or rejection of the SM. On the one hand, the SM has quite a few internal and external deficiencies that have motivated the creation of alternative models going beyond it (BSM). On the other hand, it is not easy to separate the signature of a SM Higgs boson from those of some BSM models. As a consequence, it took a period of about three years, from the initial evidence for the new particle in 2011, to 2013 and the measurement of its key properties, before the community was convinced that "The Boson" was indeed a Higgs boson. Notwithstanding the extended process of confirmation, these developments quickly led to a change in experimental strategy. Physicists have tentatively assumed that they have discovered the SM Higgs boson while simultaneously scrutinizing it for possible non-SM properties.

In order to study this development empirically, our paper avails itself of two peculiar characteristics of LHC physics. First, there are semi-annual conferences that feature reports on the recent comprehensive data analyses of the LHC experiments, including assessments of the status of various theoretical subfields. In these conferences, an experimentalist and a theorist are each tasked with giving a summary report which includes presenting and evaluating the status of the experimental evidence concerning the Higgs and provides a list of open problems. These conferences and summaries provide us with an authoritative view of the physicists' own assessment of the milestones of Higgs confirmation. These included not only the $5 \sigma$ threshold separating excess events (or evidence) from discovery (or observation), which was crossed July of 2012, but also the milestones set for confirming that the particle was a SM Higgs boson, e.g., the particle's spin and parity. In Section 2, we present a brief history of these milestones and show the increasing confirmation of the particle as a SM Higgs boson.

Second, there is a single storehouse for research in particle physics: before publication, all papers are deposited in their respective categories on the e-print archive, arXiv.org. A quantitative keyword search covering the electroweak symmetry breaking (EWSB) sector of particle physics has allowed us to assess the model dynamics of the field. We present the results in Section 3 for the presently most popular model-groups of EWSB.

Our case study bears interesting lessons for the philosophical discussions about confirmation and model dynamics. In Section 4.1, we present basic criteria for comparing degrees of confirmation and expose some of the limits of a purely logical approach to understanding the reception of the discovery as a victory for the SM. There is, of course, a strong case to be made that the Higgs boson discovery supports and highly confirms the SM hypothesis for EWSB, due to the precision and novelty of the SM predictions. But the confirmation of the SM does not stem entirely from direct support, but also from indirect confirmation through a lack of evidence for BSM physics.

In Section 4.2, we examine one of the surprising features of our data: the longevity of SM alternatives, even in the face of evidence that seems disconfirming. We understand this persistence by borrowing the Lakatosian concept of a research programme, where the core idea behind a group of models survives, while accommodating incoming data, even if that data squeezes particular models out of the available parameter space in the long run. Thus, BSM model-groups can survive, depending on their empirical and theoretical performance, both internally and with respect to their competitors. We will demonstrate the adequacy of this approach with a case study, the dynamics of Composite Higgs $(\mathrm{CH})$ models. 


\section{Steps Towards the Higgs Discovery}

This section provides a short historical overview of the milestones in the discovery process that ultimately led to the confirmation of the SM Higgs boson. It also provides context for the data we have collected on the frequency of preprint archive entries for various models of EWSB, which in turn provides the empirical material for our philosophical discussion.

To begin, we will briefly discuss the SM Higgs boson, its properties, and its importance in particle physics. We will then examine the developments that led to the announcement of a Higgs-like boson in July of 2012. This is followed by an overview of the additional evidence that led the physics community to accept that LHC had indeed discovered not only a boson that was Higgs-like, but a Higgs boson that is SM-like (Bhattacharya and Jain, 2016, see e.g.). We will end with a short discussion of the most pressing question remaining after the Higgs boson discovery: what avenues remain to rule out its potential BSM character?

\subsection{The SM Higgs}

The Higgs mechanism was first posited in the 1960s (Englert and Brout, 1964; Higgs, 1964a,b; Guralnik et al., 1964). It leads to four fields, three of which give masses to the (electro)weak bosons; the fourth becomes an observable particle: the SM Higgs boson. The Higgs boson quickly became a cornerstone of the SM after it was shown that it makes the electroweak theory renormalisable ('t Hooft, 1971).

Within the SM, the Higgs boson is an elementary particle with unambiguously predicted quantum numbers, notably its spin $\mathrm{J}$, parity $\mathrm{P}$, and charge conjugation parity $\mathrm{C}$ being $\mathrm{J}^{\mathrm{PC}}=$ $0^{++}$. While theory required its mass to be smaller than about $800 \mathrm{GeV}$, the exact value could not be predicted. However, Higgs production and decay properties are precisely known as a function of mass (see e.g. (Dittmaier et al., 2011)). This allowed for a clear experimental search strategy. The LEP $e^{+} e^{-}$collider, operating in the 1990s, and later the Tevatron $p \bar{p}$, reached sufficient experimental sensitivity to begin Higgs hunting. They did not find a signal, but excluded a SM Higgs with a mass below $114.6 \mathrm{GeV}$, and within a small interval around $160 \mathrm{GeV}$, respectively. Furthermore, under the assumption that the SM was correct, and using precision measurements and calculations of virtual effects, it was found that the Higgs should have a mass below 156 $\mathrm{GeV}$.

Alternative extensions of the SM had been developed in answer to its internal shortcomings, like the number and structure of fundamental matter particles and their respective interactions, as well as external deficiencies, like having no dark matter (DM) candidate. Many of these BSM models explicitly addressed EWSB. These include supersymmetric (SUSY) variations, CH models, models with an extended Higgs sector (e.g. assuming an additional doublet), and even Higgsless models. The main goals motivating the construction of the LHC were to probe the multi-TeV range for BSM effects and, in particular, to understand the mechanism of EWSB. It began operation at a collision energy of $7 \mathrm{TeV}$ in March 2010, collecting data until the end of 2012, by which it had increased to $8 \mathrm{TeV}$. Since 2015, the LHC has been collecting data at 13 $\mathrm{TeV}$.

\subsection{Late 2011: Promising Excess}

After continuously extending the excluded mass range for a SM Higgs boson, the ATLAS and CMS Collaborations presented their preliminary results of the 2011 data taking at a joint seminar at CERN on December 13, 2011. Both ATLAS and CMS showed that they could exclude almost the entirety of the possible Higgs mass range except for $115-130 \mathrm{GeV}$ and very high masses of 
more than $450 \mathrm{GeV}$ (ATLAS Collaboration, 2011; CMS Collaboration, 2011). More importantly, they independently reported an excess of events around $125 \mathrm{GeV}$ in both the $\gamma \gamma$, or di-photon, channel and the $\mathrm{ZZ}^{*} \rightarrow 4$ lepton channel. ${ }^{1}$ Each collaboration obtained a local significance ${ }^{2}$ of about 2.5-3.5 $\sigma$, which is not enough to claim an observation. ${ }^{3}$ But because the yields (the modes and strengths in which the excess was observed) and mass were consistent with the SM Higgs prediction, there was a strong expectation in the physics community that a Higgs discovery was in reach as more data came in.

Early in 2012, the data had been analysed in more detail and the searches extended to additional potential Higgs decay modes, essentially confirming the previous evidence (ATLAS Collaboration, 2012a; CMS Collaboration, 2012a). ATLAS and CMS, along with the CDF and D0 experiments from the Tevatron, presented their findings at the XLVIIth Rencontres de Moriond in March of 2012. In that conference's experimental summary, Dissertori, called the excess "tantalizing at this stage". However, he warned that "we should not forget the still limited statistics available," nor the "still not overwhelming significance of these observations." He concluded by remarking that "the final missing building block, the Higgs boson, [is] probably on the horizon" (Dissertori, 2012).

\subsection{July 2012: Observation of a Higgs Candidate}

On July 4, 2012, on the eve of the $36^{\text {th }}$ International Conference on High Energy Physics (ICHEP) in Australia, the ATLAS and CMS collaborations presented the result of their Higgs searches at a special colloquium at CERN. Based on an increased data set taken at the higher energy of 8 $\mathrm{TeV}$, the two collaborations reported an excess at $125 \mathrm{GeV}$ with a local significance of $5 \sigma$, the minimum significance that is conventionally considered sufficient for declaring an observation of a new particle. The significance was driven by the precision channels of $\gamma \gamma$ and $\mathrm{ZZ}^{*} \rightarrow 4$ leptons. The observed mass, along with preliminary examinations of the branching ratios, fit within the expectations of a SM Higgs boson. These data, along with analyses of additional decay channels, were submitted to journals at the end of July (ATLAS Collaboration, 2012b; CMS Collaboration, 2012b).

Both collaborations were careful to state that, though the particle's known properties were consistent with a SM Higgs, further data would be required to determine if the last missing SM particle had been found. In fact, much of the talk of the conferences following the observation announcement was dedicated to analysing the available data for clues to the exact nature of this new particle and for any signs of SM deviations. One of the most pressing questions was whether this new particle was a Higgs "impostor", such as a composite Higgs. For instance, Pomarol discussed the implications of the data for various Higgs models in his EWSB summary at ICHEP 2012, noting that the observation likely spells the end of Higgsless models and that the observed mass is just barely consistent with the ranges allowed by $\mathrm{CH}$ models or the minimal supersymmetric model (MSSM). With the question of whether or not the particle is a SM Higgs or an indicator of BSM physics looming large, Pomarol assured the conference that a new era of physics has just begun (Pomarol, 2012).

At the 2012 Higgs Hunting Workshop, Peskin outlined the steps required to establish whether the particle was a Higgs boson: (i) determining that it is a CP-even spin-0 field, (ii) identifying that the major decay modes are present, and (iii) confirming that their signal strengths are SM-like. He also outlined (iv) the need to search for small SM deviations, because precise Higgs

\footnotetext{
${ }^{1} \mathrm{~A} \mathrm{Z} \mathrm{Z}^{*}$ is an off-shell $\mathrm{Z}$ boson with a mass significantly different mass from the default one.

${ }^{2}$ In contrast to a 'global significance', this does not account for the look-elsewhere effect.

${ }^{3}$ See (Franklin, 2013) for the criteria particle physicists use to claim 'evidence' versus 'observation' or 'exclusion'.
} 
measurements may be the best route for finding new physics. Although Peskin noted that some of the observed relative strengths of the branching ratios, particularly in the di-photon channel, were suggestive of a SM deviation, by mid-2012 there was simply not enough information to make a clear determination (Peskin, 2012).

As we can see, in the first days and weeks after the observation announcement, there was a great deal of speculation but no firm answers about the exact nature of this new particle. Though the data leading to the announcement were consistent with a SM Higgs of $125 \mathrm{GeV}$, room remained in the model landscape for SM extensions that could produce similar results. This led to the common description of the particle as a "Higgs-like" boson. In this environment, many physicists working on BSM models moved to incorporate the available data, which were quickly being analysed for tell-tale clues indicative of the particle's precise nature.

\subsection{Late 2012-Early 2013: From Observation to Higgs}

Five months later, in November 2012, participants at the Hadron Collider Physics Symposium in Kyoto, Japan, and a satellite workshop on Higgs Coupling in Tokyo, assessed the current status of the observed boson. By then, up to twice the amount of data used for the July announcement had been analysed by various studies. New results were presented on the particle's couplings to fermions and bosons, to a large extent derived from loop processes. In addition, a first direct indication of the Higgs coupling to fermions $\left(H \rightarrow \tau^{+} \tau^{-}\right)$had been seen, albeit at just $1 \sigma$ significance. Furthermore, the $0^{+}$property of the SM Higgs boson agreed with data, while the spin J-parity $\mathrm{P} J^{P}=0^{-}$was found to be disfavoured. This supported the idea that the new boson was a CP-even spin-0 field, corresponding to step (i) mentioned in the previous section.

The general mood was that the incoming data was in agreement with the SM Higgs, and so the observed boson did not herald BSM physics. For example, Mangano stated in his summary that, while there is still the possibility for new physics within these discrepancies, "new physics is not jumping at us" (Mangano, 2012). Similarly, Grojean concluded that "the value of the Higgs mass together with the absence of any additional new physics so far restrict any BSM model to exotic corners of its parameter space" (Grojean, 2012). Camporesi looked to the future, declaring that "[w]e are the last generation of Higgs Hunters." He metaphorically summarized the status: "It smells like fish, looks like sushi, taste[s] like sushi and it comes with wasabi and ginger...so it is probably sushi." While acknowledging the possibility of eventual deviations, he suggested a rather pragmatic way forward, recommending the assumption of a $125 \mathrm{GeV}$ SM Higgs when calculating comparisons and p-values going forward (Camporesi, 2012).

In the five months after the observation, the mood of the larger particle physics community had significantly changed, leaning more and more towards identifying the observation as the SM Higgs. This became apparent at the Moriond sessions of March 2013. By then, the whole data set of the first run of the LHC (before a two-year shutdown) had been analysed. This analysis consisted of almost four times the $8 \mathrm{TeV}$ statistics available on July 4, 2012. Although tentative, the LHC experiments had found important new insights. The measurements of the decay into gauge bosons were consolidated and no deviation had yet been found. In addition, the indication of the $H \rightarrow \tau^{+} \tau^{-}$decay became stronger, although still not reaching the level of $3 \sigma$ necessary to claim as 'evidence'. Complementing these measurements of SM decays were searches for suppressed non-SM decays that would have hinted at BSM phenomena.

Indeed, the results indicated that the new boson was special. Whereas all previously known bosons couple universally to all fermions, i.e. with (almost) the same strengths, significantly, their couplings to the Higgs boson are vastly different, as underscored by the absence of any decay in $\mu^{+} \mu^{-}$in comparison to the yield of the $\tau^{+} \tau^{-}$decay. Instead, the coupling strengths seemed to show the mass dependence expected for the SM Higgs. Similarly, the observed pattern 
of couplings $g$ to a pair of gauge bosons $\gamma, Z$, and $W$

$$
g_{\gamma \gamma} \ll g_{Z \gamma} \ll g_{W W} \sim g_{Z Z}
$$

is in line with the expectation of a SM, mass-dependent Higgs coupling (Mangano, 2013). In addition to these measurements, the $J^{P}=0^{+}$assignment became even stronger. These measurements represented the second milestone.

Mimicking Camporesi's Tokyo summary, Sphicas declared that the new particle "is beginning" to look, sound, smell, taste, and feel like the SM Higgs, though he is careful to note that it could "have several non-SM properties and still be the Higgs boson of electroweak symmetry breaking" (Sphicas, 2013). In a similar spirit, Zwirner, in his theoretical summary of the EW session, suggested that, because the properties of "The Boson" (as he called it) are in line with the SM Higgs, "we are finally ready to change our introductions to particle physics courses..." He reiterated, however, that there are still many ways for The Boson to fail to match the SM (Zwirner, 2013).

Denisov concluded his experimental summary of the Moriond QCD session by saying that the "Standard Model stands stronger than ever" (Denisov, 2013). Mangano provided the theory summary, emphasising the strong indication of the role of the boson for EWSB, claiming that he is "not aware of any compelling... alternative framework." On the other hand, he emphasised that, "[g]iven the large overall uncertainties, nevertheless, there is clearly room for deviations to be detected." On the whole, he gave a positive answer to the question of whether the particle is $a$ Higgs boson, but remained neutral on the question of whether it is the Higgs boson (Mangano, 2013).

Finally, according to the CERN press release for Moriond 2013,

CMS and ATLAS have compared a number of options for the spin-parity of this particle, and these all prefer no spin and positive parity. This, coupled with the measured interactions of the new particle with other particles, strongly indicates that it is a Higgs boson. (O'Luanaigh, 2013)

This attitude is demonstrated in the shift in their contributions of the official status of the particle from a Higgs-like boson to a SM-like Higgs boson. Even with this shift in terminology, physicists are careful to state that it is still an open question whether or not the particle is the Standard Model Higgs boson. Answering this question requires much more data on the particle's branching ratios.

\subsection{The State of the Higgs Today: Living Well Despite Insecurity}

At the end of 2013, particle physics entered a stable situation concerning the Higgs boson. Although physicists continued to scrutinize the Higgs boson and deliver new, more precise and comprehensive studies, progress slowed down. Searches for deviations from the SM Higgs signal proceeded, but none have yet been observed, even with the increased data collection available after the LHC's $13 \mathrm{TeV}$ upgrade in 2015. The particle observed in 2012 is treated as the boson of EWSB, and in most cases as specifically the SM Higgs.

Prospective studies have been made about how much more precisely the properties of the Higgs boson could be measured at the LHC, with the increase expected to be a factor of 20 by 2022 and an additional factor of 10 by 2035. Broadly speaking, the measurements of the coupling strengths of the Higgs to $W W, Z Z, \gamma \gamma$ should improve by a factor of $2-3$ and be known a precision of $\pm 10 \%$. Physicists expect to see greater improvement in the couplings to fermions, $H b b, H \tau \tau$. In addition, the important $H t t$ coupling will be measurable directly, instead of being indirectly inferred from quantum loops, as it is today, though these coupling strengths 
will only be known to some $25 \%$. Another fundamental aspect of exploring the Higgs mechanism is to measure its potential via the three-Higgs self-coupling, $h h h$. Physicists at the LHC should eventually be able to observe this process, but the precision is likely to be fairly weak.

In summary, although substantial improvements on the measurements of the Higgs properties can be expected, even within the next 20 years, room for deviations from the SM will remain, because deviations may be hidden, even from the LHC's most sensitive searches.

\subsection{Resolving the Nature of EWSB by BSM Signals}

As previously mentioned, many alternative models of EWSB have been developed. The dominant alternatives are supersymmetric variations, $\mathrm{CH}$ models of various forms, and other models that assume that the Higgs sector is more complicated than just one $\mathrm{SU}(2)$. All these models have observable effects in the couplings, and sometimes allow only a certain mass range, but all of them predict additional particles. Many concrete realisations of these models lead to relations between the Higgs mass, its couplings, and new phenomena. With the more precise measurements of the Higgs sector that are being achieved, the parameter space for BSM predictions is being greatly reduced.

SUSY models need at least two Higgs doublets, leading to five physical scalar particles instead of one. It is a firm prediction that the lightest of these should have a mass of less than 130 GeV. ${ }^{4}$ In its minimal version (constrained MSSM, or cMSSM), the masses of the scalar particles are strongly related to four parameters, but even in the cMSSM observing the decays may be complicated. On the other hand, there are as many new supersymmetric particles predicted as ordinary particles in the SM, and at least some should be detectable in the range accessible at the LHC.

Similarly, additional Higgs bosons are predicted in more complicated Higgs sectors, even without embedding such a sector in a larger theoretical framework. Examples include two Higgs doublet models, but also those adding a Higgs singlet. Such extensions are less constrained than their supersymmetric counterparts. They predict extra Higgses, but no additional particles outside the SM.

Composite Higgs models come in various forms. The original idea copied the structure of the strong interaction, which led to the Technicolor model. Some variants can accommodate the properties of the LHC boson-although the parameters must be stretched. All CH models expect new particles to appear in the $\mathrm{TeV}$ range, especially partners to the top quark. $\mathrm{CH}$ models will be discussed in greater detail in Section 4.2.2.

What all these models have in common is that they predict both variations in the Higgs couplings and new, non-SM particles. Finding these features at the LHC would revolutionize particle physics. For example, a more complicated Higgs sector would imply the existence of more scalar particles, probably at higher masses, while new heavy particles at the $\mathrm{TeV}$ scale would imply CH models. Detecting these might be possible at the LHC, but is not guaranteed, even if there is something to be found. The situation may stay ambiguous for the foreseeable future. $^{5}$

One possible way forward are the model-independent descriptions of deviations from the SM that are becoming more and more popular among theorists. One approach is to use effective field

\footnotetext{
${ }^{4}$ This parameter space is considerably smaller than that of the SM, which makes it a bolder and more precise prediction, though small increases of the limit are possible (for example, the Higgs mass would increase logarithmically if supersymmetric particles were significantly higher than $1 \mathrm{TeV}$ ). These bolder predictions have interesting implications for the confirmation of these hypotheses, which we will discuss further in Section 4.1.2.

${ }^{5}$ The construction of a linear $e^{+} e^{-}$collider (ILC) is being considered and could lead to an improvement of the coupling measurements by some factor of 10 , but it is unclear when it would become operational.
} 
theories (EFTs) and make operator product expansions (OPEs) of possible processes that essentially parametrize physics properties while allowing BSM contributions. These do not predict new particles per se, but in case of observed deviations, they can be used to indicate potential deviations in other observables.

The epistemological questions then are: how should physicists operate in this situation; and how much justification exists to work with the observed object as if it were the Higgs boson? In other words, what is the proper approach to this double-life? We will turn to this in Section 4.

\section{Model Popularity on ArXiv}

We now come to the project's second avenue of research. In this section, we examine the popularity of six major groups of models in the EWSB sector using the number of papers submitted to the arXiv via its INSPIRE keyword system. This avenue is focused less on the discovery of the Higgs boson itself and more on the effect that the discovery process has had on BSM accounts. Based on the above analysis of the announcements and summary papers, it certainly seems as though alternative models of EWSB are in trouble. Looking at the changing number of papers on these alternative models allows us to see how the landscape of models has been affected by the LHC data. In this section, we approach model-groups from an operational standpoint and represent them in terms of a string of keywords, which we have listed in Appendix A. These groups are the ones listed in the previous section. Most of the model-groups are composed of models in the sense of today's philosophy of science, but given our operational approach in Section 3 , we do not require this at this point. In Section 4.2, we will present a philosophical basis for understanding the nature and role of these diverse model-groups.

ArXiv.org is an online archive of e-prints for research papers in physics, mathematics, and various related disciplines. Papers are uploaded and made available daily and this allows us to track changes in research interests as they develop, giving us a quantitative way of looking at the development of the SM Higgs in comparison with various alternatives.

\subsection{Method}

\subsubsection{INSPIRE Keyword System}

ArXiv's INSPIRE keyword system automatically tags uploaded papers with specific keywords when it finds matches or known synonyms. If the abstract or title of a paper makes use of a phrase such as "two Higgs doublet model," it will be tagged with the keyword "Higgs particle: doublet: 2." Searching only the title and abstract is intended to prevent "noise" resulting from a full text search, as authors tend to mention what they are not doing. A single mention is enough to generate a keyword tag. Keywords can also be manually added by the author when uploading the paper.

\subsubsection{Code}

In order to perform the searches in a reasonable timeframe, a Python script was written that called the INSPIRE website, http://inspirehep.net, to build a dictionary of the search results using the website's SPIRES-style syntax. The code then reads the dictionary and counts the length of (number of entries in) the dictionary that match the dates of interest. The number of hits for the search term are then printed and collected in a spreadsheet. 


\subsubsection{Organising the Data}

The searches were conducted for the six most currently popular model-groups: Composite Higgs/Dynamic Symmetry Breaking Models; Supersymmetrically-Extended Higgs Models; NonSupersymmetrically-Extended Higgs Models; Higgsless and Extra-Dimensional Models; ModelIndependent (EFT and OPE) approaches; and the Standard Model Higgs. ${ }^{6}$ The string of keywords defining each model-group reflect the categorizations used by the physicists themselves. Each model was also separated according to its 'primary archive' (as it is referred to on the arXiv), which is determined by whether it is hosted on HEP-EX or HEP-PH (which denotes experimental and phenomenological/theoretical entries respectively). The primary archive function was used to avoid double counting papers that are cross-listed to other archives.

The results are presented as the average number of papers per month over a six-month period, from January 2010 to December 2017. For present purposes, the results of interest are those that show the large scale trends in the popularity of model-groups.

\subsubsection{Avoiding Potential Issues}

Negative Mentions Some keyword tags will be negative mentions of a model. There is little that can be done to filter this without doing a complex full-text analysis. Nonetheless, the fact that a model is mentioned, even if only to show that it doesn't fit the data, or to have its parameters further constrained, is still an indication of its popularity. This data is not intended to be a representation of physicists estimates of the likelihood of a model's success.

Artificial Fluctuations It is worth mentioning a few facts that may create small fluctuations in the data. First, some conferences are held specifically to discuss some models and not others. For instance, there are regular conferences for charged Higgs and for supersymmetry, but not for composite Higgs. This is still an indication of the popularity of a model and the number of people willing to work on and research it. In any case, the numbers of conference papers, while not insignificant, do not appear to vary wildly from model to model. All papers are categorized by document type, using what the arXiv calls "type-code". These can be used to separate conference proceedings from theses, books, refereed journal articles, and so on. For most searches, the number of refereed journal articles constitute roughly $60-80 \%$ of the total keyword numbers, $10-15 \%$ for theses, $10-20 \%$ for conference presentations that are not also published articles, and a few percent for books, review articles, lecture notes, and other. Aside from conferences, the LHC was shut down for upgrades from 2013-2015, leading to stretches of data-taking that may produce artificial bumps in the plots. The effects of conferences and data-taking periods are mitigated by presenting only the number of models per month over a 6 -month period, effectively smoothing over these small bumps.

Of course, in the beginning of 2016, there was a great deal of excitement in the physics community about an excess in the di-photon channel at $750 \mathrm{GeV}$ in both ATLAS and CMS of around $3 \sigma$ significance. This led to a great deal of theorizing and publication on the nature of this potential particle within models that predict additional particle content. At increased luminosity, the bump disappeared in the statistics. We will not focus on this event, other than to say that it had an effect on the number of papers from various BSM models in 2016.

Double Counting One potential concern is that because all the papers have several keyword tags, some models may be counted twice and inflate the seeming popularity of the model. There

\footnotetext{
${ }^{6}$ Because there is no single keyword term for the SM Higgs, it has been estimated using keywords for experimental results and measurements on the Higgs boson doublet which do not feature supersymmetry, dark matter, or charged or composite Higgses (see Appendix A).
} 
are two different ways in which this may occur: a) a paper containing multiple tags belonging to the same model-group; or b) a paper containing tags belonging to different model-groups.

Within a Model-Group The first potential for double counting may occur when a model features several closely related keywords such as: "supersymmetry," "minimal supersymmetric standard model," "supersymmetry: minimal," and "Higgs particle: doublet: 2." These four keywords may appear on a single paper concerning a single model, but it will result in four keywords being tagged, thus inflating the total numbers of papers working on supersymmetric models. However, the total number itself is not shown in our data. Rather, our comparison of model-groups is done with a long-string Boolean search term composed of ANDs, ORs, and AND NOTs, such that a single paper with four supersymmetric keywords counts only once in the model-group total. The number of keywords are not merely summed to achieve the model-group totals. This kind of double counting should be minimal. For the full list of search terms used, see Appendix A.

Between Model-Groups The second potential for double counting may arise when a single paper has keyword tags from two different model-groups. This may occur when a paper takes a hybrid approach to EWSB, or merely mentions multiple approaches in the abstract or title. For instance, searches for charged Higgs particles could be a part of a supersymmetric extension or a non-supersymmetric extension. This is mitigated by separating papers that have a charged Higgs tag into those that also have a supersymmetry tag and those that do not. They are organized accordingly in the model-groups. For example, searches for a non-supersymmetric charged Higgs make use of explicit search term "and not ( "supersymmetry*" or "minimal supersymmetric standard model")". This ensures that the model is only counted as a nonsupersymmetric extension of the SM. On the other hand, the search for a supersymmetric charged Higgs does just the opposite. The same was done for many models that could be under the frameworks of different model-groups, such as the two-Higgs doublet. This is mostly relevant for comparisons of individual models which are not the focus of this paper.

\subsection{Model-Group Search Results}

In this section, two plots of model-group popularity over the last eight years are presented. The plots are displayed in terms of the number of papers per month for each search term, averaged over 6 -month periods. The data is shown beyond the Higgs discovery period outlined in Section 2 in order to set a baseline before the LHC data analysis and show this period as a part of longer trends. The 6-month average allows us to smooth over artificial peaks and troughs, as discussed above. Figure 1 shows papers tagged as experimental; those tagged as phenomenological/theoretical are shown in Figure 2. The total number of relevant papers in the HEP-EX archive was 612, and the number in the HEP-PH archive was 4485. These two plots provide quite different pictures of the changing landscape of models over the years.

As shown in Figure 1, the overwhelming experimental activity was related to studying the boson in terms of the SM. The strong rise in SM papers in late 2017 is due to the recent $13 \mathrm{TeV}$ data analyses. One should bear in mind that any determination of the Higgs' properties is simultaneously a test for possible BSM deviations. To a lesser degree, there are also explicit searches for additional Higgs bosons in the framework of supersymmetry or more complicated Higgs sectors. Finally, there is a slight increase in studies using OPEs that is apparent within our timeframe.

For theory papers (Figure 2), we see that SUSY is the most popular model-group throughout the past eight years. From 2010 to 2013 the group almost doubled. This model-group 


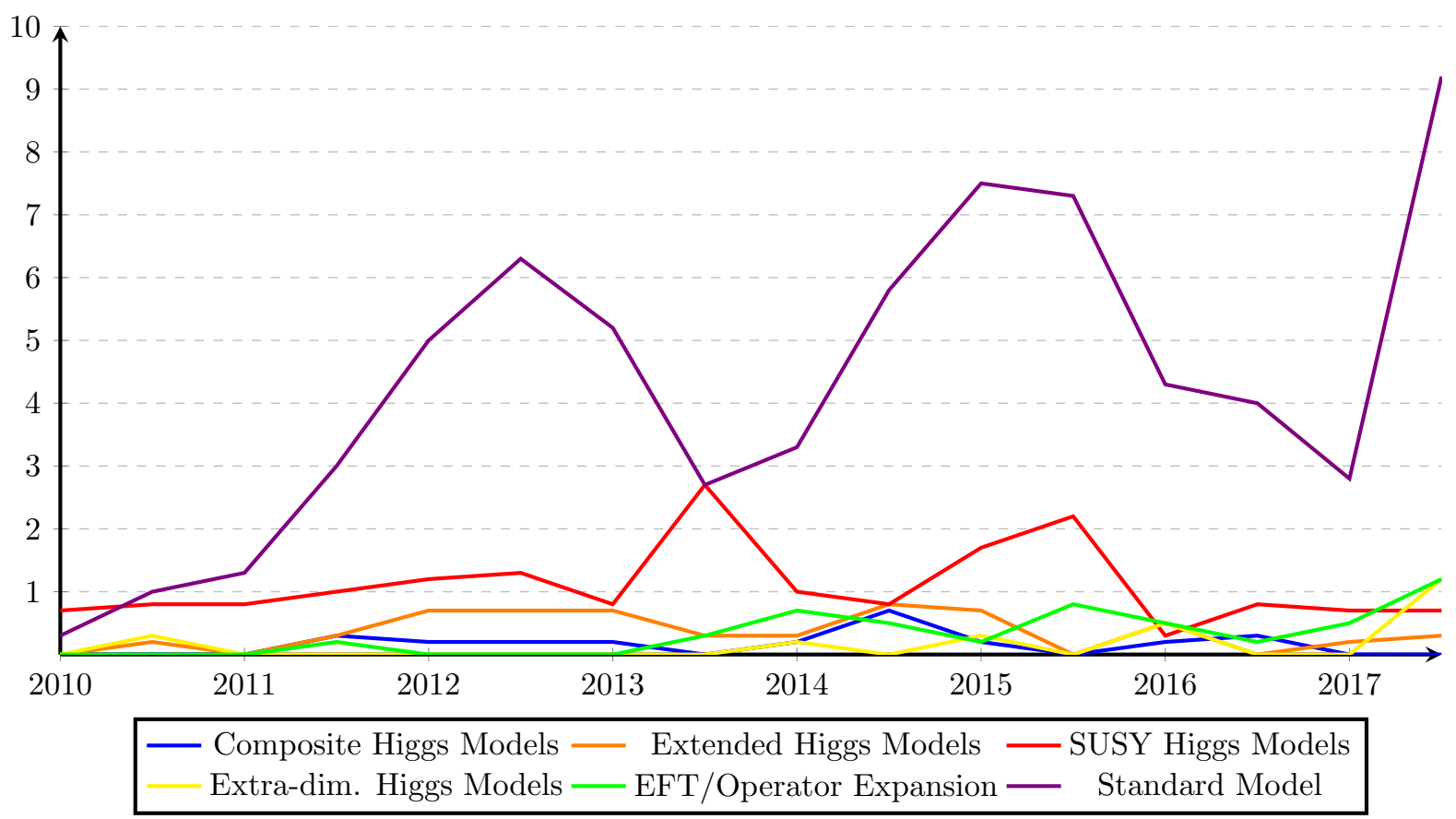

Figure 1: Overview of Model-Groups(HEP-EX) from Jan 2010-Dec 2017

peaked in late 2013, and aside from a bump in early 2015, has been steadily declining since. Non-supersymmetric extensions have quickly gained popularity, largely due to the nonsupersymmetric 2-Higgs doublet model. However, this should come as no real surprise, since an additional doublet is the simplest extension of the Higgs sector. EFT and OPE approaches are also steadily growing in popularity, increasing almost tenfold over the eight years we observe. $\mathrm{CH}$ models, extra-dimension and Higgsless models are, quite surprisingly, fairly steady over the eight year period, though showing a recent decline. Theoretical studies on the SM Higgs remained fairly constant, primarily focusing on improving the precision of the calculations.

There are two interesting messages that the search data conveys. First, even though EFT and OPE approaches began showing up in 2010, there is a strong increase in their popularity beginning in 2013. This rise may reflect an increased instrumentalism about BSM models, driven most likely by the lack of evidence for any BSM physics. This approach has emerged as an alternative to the direct searches for BSM models, which have yet to turn up anything promising. The incoming evidence for the SM and the remaining popularity of alternatives is an interesting phenomenon we will examine through the lens of confirmation (Cf. Section 4.1).

Second, even with the Higgs discovery, other models still persist and sometimes increase in popularity, at least initially. For some models, the Higgs sector is merely an extension of the SM Higgs, so it is no surprise that they can easily accommodate a Higgs boson with these properties. But more interestingly, other models make use of very different mechanisms that feature no physical Higgs boson. The number of papers per month for extra-dimensional models remains quite low, and has only very recently decreased. The same is found with models of dynamical, or strong, symmetry breaking. It is sufficient here to mention that a "squeezing out" of models, a disconfirmation, happens on a longer timeline than one might have thought. We will attempt 
Model-Groups HEP-PH

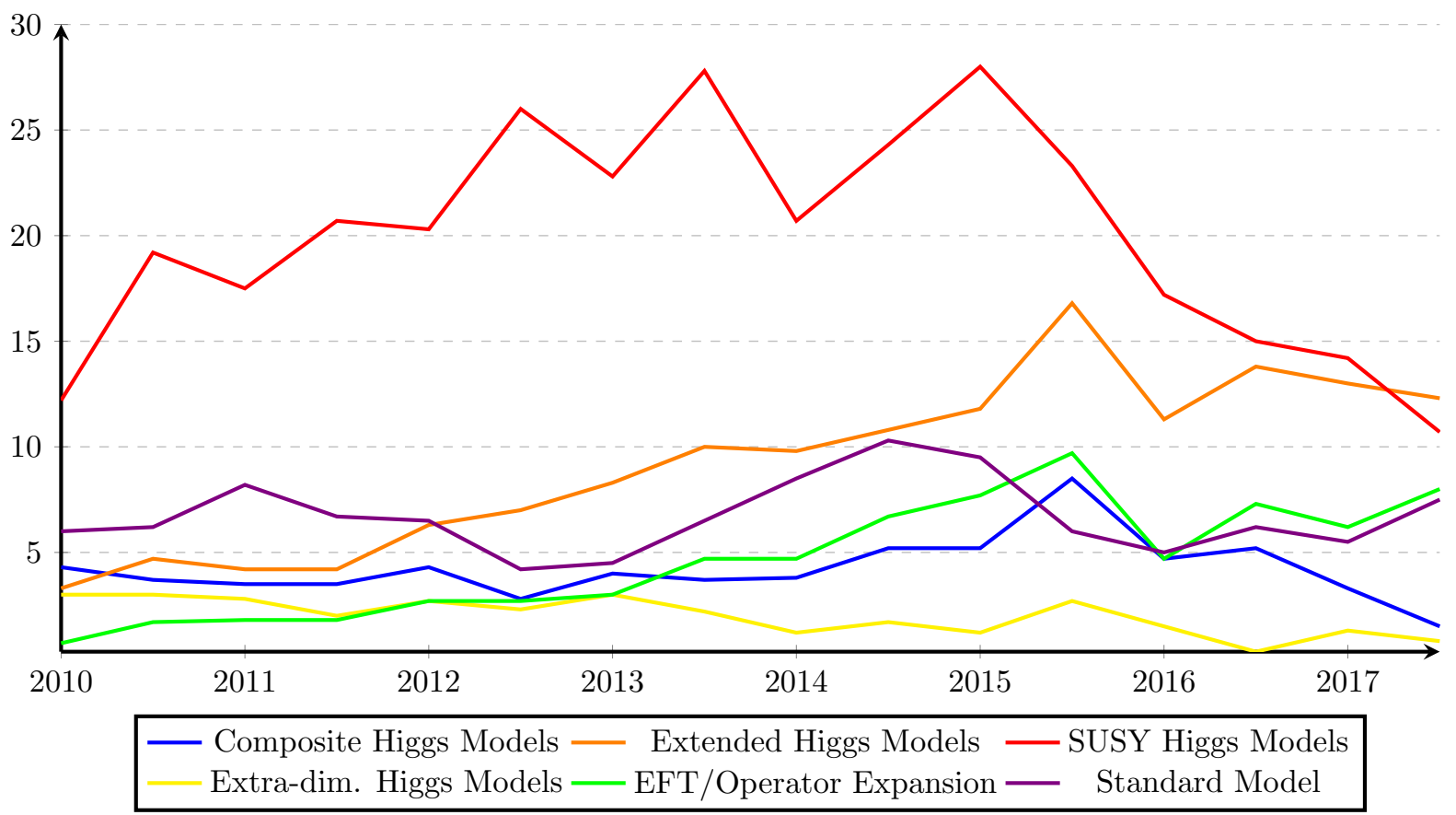

Figure 2: Overview of Model-Groups (HEP-PH) from Jan 2010-Dec 2017

to explain this phenomenon in the following section by analysing what is revealed about the way confirmation works in particle physics and by providing a framework for model dynamics.

\section{Discussion}

Our detailed study of the Higgs discovery allows us to examine the process by which scientific hypotheses are actually confirmed or disconfirmed, and gives us guidance in studying the dynamics of the model landscape. What we find is that the practice of confirmation is only partly revealed through the logical connections of data and hypothesis, which we explore in Section 4.1. The SM EWSB hypothesis has been strongly confirmed by the precision of the data and the novelty of the prediction. However, from the standpoint of the logic of confirmation, the SM is not the only hypothesis to have been increasingly confirmed. When using the same measures, some alternatives, like minimal supersymmetry, are found to be confirmed by the observation to an even higher degree. The attitudes of physicists with respect to confirmation, as presented in Section 2, do not reflect this, but rather reveal the interplay of direct and indirect confirmation of hypotheses that influences their acceptance of certain model-groups. We will expose some of the limits of the logic of confirmation to account for the actual and perceived evidential development of a hypothesis in practice.

One of the surprising aspects of our study is the persistence of alternative models of EWSB throughout the discovery and confirmation process, as mentioned in Section 3. In Section 4.2, we offer a framework to understand the temporal development, or dynamics, of the model-groups comprising the EWSB sector. We borrow Lakatos's notion of a research programme to show how 
the "hard core" of a model-group can survive, preserved by a "protective belt" that adapts to new experimental and theoretical inputs. These hard cores feature elements that physicists find attractive and worth defending, providing the stable foundation for model-groups to shift and accommodate to seemingly disconfirming circumstances. This explains why BSM models are not refuted, but rather squeezed out.

\subsection{Confirmation of the SM Hypothesis}

Let us begin by outlining some general features of confirmation and emphasising that confirmation happens by degrees. To say that some hypothesis $H$ is confirmed by evidence $E$ is merely to say that $E$ increases the degree of confirmation of $H$, and does not imply that $H$ is now, because of $E$, known to be true. Similarly, when $E$ lowers the degree of confirmation of $H$, it is said to disconfirm $H$. Theories and hypotheses are confirmed to various degrees by the amount and variety of the evidence that supports them (see Hempel, 1965). $H$ will be highly confirmed by its evidential base $E$, if $E$ is broad, varied, and precisely predicted. We are considering a fixed set of evidence, so we will focus on precision and novelty of predictions rather than variety of evidence.

In the broadest sense of the term, we can say that $E$ confirms $H$ if:

1. $E$ is a novel prediction of $H$

We include the term 'novel' here to ensure that $E$ is a genuine prediction of $H$ (see Howson and Urbach, 1991). Further, confirmation is a matter of degrees, and it is reasonable to hold that $E$ strongly confirms $H$, or confirms $H$ to a high degree, if:

2. $E$ is a precise prediction of $H$

Novelty and the riskiness of predictions have long been regarded as important for the confirmation of scientific hypotheses (see, e.g., Popper, 1963; Lakatos, 1978; Mayo, 1991, 2000). We are much more inclined to think that a hypothesis gets something right if it makes novel predictions than if it only accommodates known evidence. This is in part because it precludes the use of $a d$ hoc auxiliaries and speaks to the ability of a hypothesis to truly predict, rather than accommodate. ${ }^{7}$ These kinds of novel predictions are widely regarded to be very important for confirmation and confidence in hypotheses, though formally demonstrating why and how these can be distinguished has been much debated (see Schurz, 2014; Campbell and Vinci, 1983).

We agree with Dawid (2017) that a Bayesian analysis can provide interesting insights into contemporary particle physics. However, we think that the problems discussed in the present paper can also be approached in a less formal way and are not automatically resolved in a Bayesian setting. Moreover, in the case we are examining, we are not determining whether a particle will be found at all, but rather we are interested in the confirmation of the hypothesis that the new particle at $125 \mathrm{GeV}$ is the SM Higgs boson, given the data from the LHC. We call this the SM Higgs hypothesis (SMHH), which we can specify as:

SMHH: There is a single Higgs doublet that realizes the Higgs mechanism.

Because we are here speaking of the logical confirmation of hypotheses, the SMHH is best understood as exclusive of BSM physics. With this kind of stop clause, if there is an extended Higgs sector, then the $\mathrm{SMHH}$ is false.

\footnotetext{
${ }^{7}$ For a more in-depth discussion of ad-hocness and the SM Higgs, see Friederich et al. (2014). They argue that the most crucial characteristic of an ad hoc hypothesis, a lack of experimental evidence, is no longer obeyed now that Higgs boson has been discovered.
} 


\subsubsection{Direct Confirmation}

The observation of a signal at $125 \mathrm{GeV}$ has to be seen against the backdrop of a highly successful and manifold confirmation of all other SM predictions, leading to a strong overall acceptance of the SM. In other words, the SM as a whole has a 'broad, varied, and precise' evidential basis. Of course, the SM confirmation was still incomplete without a mechanism for mass generation. However, the expectations for the Higgs mechanism was fairly high (see ?). The SMHH also makes precise and novel predictions: once the mass of the Higgs candidate was known, its other properties were precisely predicted. There was some freedom with respect to its mass and that is something to which we will return. In addition, the SMHH predicts something novel: a fundamental spin-0 particle. ${ }^{8}$ Until the 2012 discovery, every observed elementary particle was either a spin- $\frac{1}{2}$ fermion, or a spin-1 gauge boson. ${ }^{9}$ Thus, the requirements listed above indicate a potentially strong confirmation of the $\mathrm{SMHH}$.

As discussed in Section 2, the discovery of the $125 \mathrm{GeV}$ particle set in motion the process of confirming the SMHH and provides a striking example of increases in the degree of confirmation of a hypothesis. In short order, many measurements on this particle were performed. While almost all of the measurements could potentially disconfirm the SMHH, a hierarchy of their importance for confirmation emerged. The initial observation of a 'Higgs-like' particle was essentially based on just its mass and a coarse agreement of its decay modes with SM expectations. The subsequent analyses of its properties led to a stronger confirmation. Of particular importance was the measurement of its spin, which, as just discussed, implied the existence of a completely new kind of elementary particle. ${ }^{10}$

The promotion to a 'SM-like Higgs' particle required (more) precise measurements of the branching ratios. For example, only Higgs decays in vector bosons had been observed at first, so finding its decays into fermions became important. However, the fermion couplings were already derived from loop corrections, assuming theoretical input. What this reveals is a differing relevance for each piece of evidence. This relevance is determined both by the importance of the evidence for the hypothesis and its experimental feasibility. This becomes apparent from the actual progress within our timeframe, and is underlined by the importance of measuring the Higgs self-coupling, which might remain technically infeasible in the next 20 years or so. ${ }^{11}$

As an upshot of this discussion, the $\mathrm{SMHH}$ can then be considered strongly confirmed if it satisfies the two general conditions for confirmation stated above: the evidence matches the precise and novel predictions of the hypothesis. The confirmation process as seen by physicists also suggests that different measurements are differently weighted. Ideally, any theory of confirmation would account for those weights.

\subsubsection{Indirect Confirmation}

There is yet another source of confirmation for the SMHH. From what physicists are saying (Section 2), part of the reason why there is increased confidence in the SM is that even with all the data from the LHC, no new physics is "jumping out at us." There is still room for new physics, but some BSM models have LHC-accessible implications and are being forced to the edges of their parameter spaces, as noted by, e.g., Grojean (Section 2.4). In terms of confirmation, there has been no evidence for any new physics beyond the SM, and this in turn increases our confidence in

\footnotetext{
${ }^{8}$ We cannot be certain that the particle is indeed elementary, but we will follow the views of physicists and tentatively accepting the confirmation of the SMHH.

${ }^{9}$ There are other scalar particles, but these are not elementary particles like the SM Higgs boson.

${ }^{10}$ The importance is also reflected in the press release quoted in Section 2.4. It is interesting to note that the measurement of the $J^{P}=0^{+}$quantum numbers were performed by discriminating against different spin-parities. However, this was not been performed in view of alternative models.

${ }^{11}$ Though this is not really considered a show stopper for SMHH confirmation.
} 
the SM. Indirect confirmation has been widely discussed (see Laudan and Leplin (1991); Jeffrey (1992); Moretti (2002)), but we are interested in a special case of indirect confirmation, namely through the disconfirmation of alternatives. Part of the strong confirmation of the SMHH must be indirect (from failing to find non-SM properties).

One potential issue with indirect confirmation is that it begins to sound like Hempel's paradox of the ravens. ${ }^{12}$ Such worries about paradoxes can be alleviated by properly partitioning the alternatives and disfavouring or eliminating them. But this is itself quite complicated in this case, given the number of alternative models and the difficulty in isolating signals. Further, given that all BSM models encompass the SM - with the notable exception of SMHH - they are, per construction, not in disagreement with any evidence (yet). This leads to difficulties relating the status of SMHH confirmation with those of BSM models. As an example we will compare the confirmation status of the SM and MSSM in the next section.

\subsubsection{Comparing SM and MSSM Confirmation by Higgs Discovery}

Because confirmation is understood in terms of degrees, it is reasonable to compare the degrees to which different hypotheses were confirmed by the Higgs discovery $E$. The conditions above may be reformulated. We can say that $E$ confirms $H_{0}$ more strongly than it confirms $H_{1}$ if:

$1^{*}$. $E$ is a novel prediction of $H_{0}$, but only accommodated by $H_{1}$

$2^{*}$. $E$ matches more accurately the predictions of $H_{0}$ than those of $H_{1}$

We will compare the SM hypothesis $H_{0}$ to minimal supersymmetry $H_{1}$. As a reminder, $H_{1}$ requires an additional doublet, resulting in five physical Higgs bosons and a possible modification of the Higgs decay couplings. In addition, in SUSY, each SM particle is predicted to have a twin, like a supersymmetric electron or top quark (spin-0), or a supersymmetric photino (spin- $\left.\frac{1}{2}\right) .{ }^{13}$

The first thing to note is that the experimental results that are so well aligned with the SM predictions are also equally well aligned with the predictions of minimal supersymmetry. This is because models like MSSM are extensions of the SM and fully encapsulate it. All the positive, or direct evidence for the SM, is evidence for the MSSM, including the evidence of the Higgs boson itself (which can be interpreted consistently with an MSSM Higgs). ${ }^{14}$ So what is the confirmation status of the MSSM Higgs compared with the SM Higgs? The SMHH was highly confirmed by the Higgs discovery, but due to the higher precision of its prediction, the MSSM Higgs hypothesis was confirmed even more so. We will now demonstrate, and offer an explanation for, this unexpected result.

Applying condition $2^{*}$, the SM theoretically established bounds on the upper values of the Higgs mass at $\lesssim 800 \mathrm{GeV},{ }^{15}$ whereas all MSSM models imply their lightest Higgs will have a mass $<130 \mathrm{GeV}$ (Kraml, 2013; Haber et al., 1997, see e.g.) - much narrower than the SM prediction. ${ }^{16}$ In other words, MSSM made a bolder, riskier prediction by providing more precise theoretical bounds on the Higgs mass, and so the evidence ought to more strongly confirm MSSM. The SM

\footnotetext{
${ }^{12}$ This paradox arises out of two principles: Nicod's Rule, which states that $R a \wedge B a \operatorname{confirms} \forall x(R x \rightarrow B x)$, and the Equivalence Rule, that confirmation of a hypothesis confirms logically equivalent hypotheses. So, because $H_{1}$ : All ravens are black and $H_{2}$ : All non-black things are non-ravens are equivalent there is no way to formally distinguish between these types of evidence.

${ }^{13}$ To be more precise: it predicts four neutral electroweak spin- $\frac{1}{2}$ particles which can mix into four 'neutralino' states.

${ }^{14}$ In a frequently used quip, proponents of SUSY claim that half of its particles have already been discovered.

${ }^{15}$ Significantly tighter constraints have been derived from precision measurements, implying a probability of a $125 \mathrm{GeV}$ Higgs to be at the 0.2 level. For the sake of the argument we will not use this in the following.

${ }^{16}$ There exists a small (logarithmic) dependence of this bound on the masses of the SUSY particles. Even if more Higgs multiplets exist, the bound would only rise to $150 \mathrm{GeV}$.
} 
and MSSM are both well confirmed by conditions 1 and 2, but MSSM fares better on condition $2^{*}$ due to the riskiness and the precision of its prediction.

This formal result disagrees with the putative empirical successes of the models (given the discovery). Proponents of the MSSM took the mass value of the Higgs boson as an argument for its continuing viability. There are further arguments in favour of the MSSM beyond the value of the Higgs mass. For example, it has a DM candidate, which cannot be found in the SM. However, even with the strong observational and theoretical points in its favour, its proponents stop short of claiming MSSM is highly confirmed. How can we account for this? We maintain that this is largely due to the experimental status of the other predictions of MSSM.

\subsubsection{SM and MSSM Confirmation Beyond the Higgs}

The key reservation for the confirmation of SUSY is that all the predictions specific to MSSM have yet to be established by the evidence. No supersymmetric twin particles have been found. Evidence of DM in connection with the production of supersymmetric phenomena is absent. ${ }^{17}$ In terms of the confirmation process, the existence of such particles is a highly relevant prediction that distinguishes SUSY from all other EWSB accounts. This underscores a requirement for confirmation theories to assign different weights to different predictions, and to consider the relevance of the predictions.

We can formalize this sort of indirect confirmation by shifting focus from the confirming effect of the discovery to the confirming effect of a negative prediction (that some evidence will not be seen). We can do this by framing a lack of new particles as a SM prediction. Defining $E_{B S M}$ as evidence of BSM physics, we can say that:

3. If $H_{0}$ predicts $\neg E_{B S M}$ and $H_{1}$ predicts $E_{B S M}$, then if $E_{B S M}$ is not discovered, then $H_{1}$ is disconfirmed and $H_{0}$ is correspondingly confirmed.

But we should be more precise, since this kind of confirmation must adjust for experimental sensitivity. In this case, the experimental sensitivity is such that a significant segment of the parameter space of SUSY models is excluded. For example, the analysis of LHC data has excluded 90-95\% of the parameter space for cMSSM (see Bechtle et al., 2016). However, there remains a large parameter space for which there is simply no experimental sensitivity. Schematically, one can split the MSSM parameter space into $P_{1}$, for which experimental sensitivity exists for at least one feature not expected in the SM, and $P_{2}$, for which no experimental sensitivity exists for any new feature.

Therefore, we modify 3 into $3^{*}$ :

$3^{*}$. If $H_{0}$ predicts $\neg E_{B S M}$ and $H_{1}$ predicts $E_{B S M}$, for which no evidence exists, then

- if $E_{B S M} \subset P_{1}, H_{1}$ is disconfirmed,

- if $E_{B S M} \subset P_{2}$, there is no change in the confirmation status of $H_{1}$.

$H_{1}$ cannot be completely disconfirmed before the whole parameter space has been examined, but as searches progress and $P_{1}$ increases at the price of $P_{2}, H_{1}$ can be disconfirmed to higher and higher degrees. This shows that confirmation and disconfirmation may well be a matter of long-term survival rather than about a single confrontation with experiment.

Therefore, not finding supersymmetric particles does not necessarily disconfirm $H_{1}$; instead MSSM has the better formal score for its more precise Higgs mass prediction and account for an additional observation not covered by the SM. This disagreement with the physicists' attitudes

\footnotetext{
${ }^{17}$ Besides, DM candidates are also predicted in other BSM models, so understanding DM better may not bolster MSSM's confirmation.
} 
described in Section 2 can be reconciled by assigning a very high weight to the (non-)evidence of the twin particles predicted by MSSM, since it is an essential and unique prediction of the MSSM that is mandatory for any strong confirmation.

One may speculate about the impact possible future discoveries may have on the confirmation status of MSSM. If another Higgs boson is found, the confirmation status of MSSM would increase, though it would still have to compete with general models of a more complicated Higgs sector. If just one MSSM-compatible supersymmetric fermion is found, it would boost the confirmation status of the MSSM significantly. In either case, SMHH would be disconfirmed.

In addition, $3^{*}$ poses an interesting question concerning the confirmation status of the SM itself. Given that there is evidence for DM, $3^{*}$ can be interpreted as providing a certain degree of disconfirmation for the SM. Physicists are fully aware that DM implies that the SM is only a complete theory within a certain energy range that may be greater than our current experimental sensitivity. However, this is not in disagreement with the physicists' attitude, as outlined in Section 2.

\subsubsection{Confirmation vs. Acceptance}

It is obvious that a strongly confirmed theory will be largely accepted in the physicists community. However, the reverse conclusion is more involved: a theory can be largely accepted even if it is not strongly confirmed. Here, MSSM provides an important example.

MSSM is accepted (to the extent that it is) from arguments that are not strictly evidence based. Instead, there are also important pragmatic and theoretical motivations, like its simplicity and explanatory promise. SUSY, aside from providing a candidate for DM, has theoretical niceties that go beyond the abilities of the SM: it naturalises the Higgs mass and allows for gauge coupling unification, among other virtues (see ). Thus, SUSY models will likely persist, helped along by their large parameter space. However, the reduced activities on MSSM calculations observed in our arXiv analysis might indicate a reduced level of acceptance, in light of long and fruitless searches for MSSM evidence. It is also pertinent that a reduction of parameter space can affect acceptance, even if it is not a matter for confirmation.

It is important to emphasise that confirmation can be a lengthy process. This is not only true for full theories like the SM, but also for particular elements, like the Higgs boson. Confirmation is essentially a backwards-facing measure of what has been accomplished to date. A logical analysis of models and model-groups is not enough to get an accurate assessment of their putative successes and acceptances. A model is also evaluated in practice by its forward-facing prospects, including its practical and theoretical virtues. In particular, this means that in scientific practice a particle like the Higgs boson can be treated as highly confirmed, even if its properties have not been completely measured and even if a program exists to further scrutinize if the properties accord with the SMHH. An understanding of the virtues and survival of BSM models requires a broader epistemological analysis of the model's historical dynamics as it confronts new data and analyses. We discuss this in the following section on model dynamics.

\subsection{Model Dynamics in the Higgs Sector}

Alternative pictures of EWSB respond to the well documented problems of the SM and offer hope for discovering new physics. Even as the properties of the new particle were verified and a consensus in the particle physics community emerged that it matches the predictions of the SM, the pluralism of the landscape of BSM models endured. Our data from Section 3 show interesting dynamics arising from this landscape. For example, new work on composite Higgs models remained steady for a long time, despite being disfavoured from lack of any direct 
confirmation. Naively, one would expect such models to diminish in prominence (or even go extinct) much sooner from the lack of empirical evidence and data contrary to the expectations of the modellers. Nevertheless, they persist and model-groups are modified in response to the shrinking phenomenological space by physicists who continue to find their core ideas attractive. We believe the framework of Lakatosian research programmes, along with a closer look at the arXiv entries in question, will allow us to move beyond this naive view to provide an account for the model dynamics of the EWSB landscape. ${ }^{18}$

\subsubsection{Research Programmes}

Lakatosian research programmes ${ }^{19}$ are formed from overlapping series of theories. Their progressiveness is graded on the expansion and corroboration of their empirical contents. A research programme has a "negative heuristic" specifying a "hard core" composed of theories, propositions, or basic concepts taken to be unfalsifiable within the programme. This hard core forms the programme's foundation and the negative heuristic requires that it remains unmodified in the face of theoretical and empirical challenges. It is surrounded by the "protective belt" of the "positive heuristic", the aspects of the research programme which adapt to these challenges. The protective belt includes the moving parts that can be changed without affecting the hard core (adjustable parameter values, auxiliary hypotheses, etc.), while the positive heuristic describes how to change the protective belt and under what circumstances. A research programme's progressiveness can only be determined with hindsight: individual confirmations of a particular prediction do not immediately warrant a theory's acceptance and disconfirming evidence does not warrant its abandonment. Lakatos emphasized that one should be patient with respect to budding research programmes and that they should always be compared with their existing competitors. Nevertheless, researchers are obliged to keep an honest score of the progressiveness of the programmes in which they work, specifying milestone experiments to severely test them. ${ }^{20}$ But patience towards a research programme is not infinite; Lakatos suggests only issuing temporary certificates of acceptance for successful programmes and limited indulgence of failing ones. This temporal element poses no problems for the SM, because particle physicists are constantly increasing the precision measurements of particles discovered earlier, but it may be an element contributing to the recent downward trend of work in BSM models.

Whereas Lakatos developed his framework to focus on 'theory,' we will expand it to accommodate the notion of 'model' as used in particle physics. Here models are taken to be independent autonomous entities that complement, or expand upon, the SM. ${ }^{21}$ Individual models may follow Lakatos' description of models as "bound to be replaced during the further development of the programme, and one even knows, more or less, how" (Lakatos, 1978, 51). But, following Morgan and Morrison (1999), they have some autonomy from theory, which we use to carve out a distinct place for model-groups. This autonomy may lie in their forms, the guiding ideas motivating their construction, or in their specific functions within particle physics. ${ }^{22}$ With our modification we

\footnotetext{
${ }^{18}$ Our application of Lakatosian research programmes to particle physics is not unique (see Johansson and Matsubara (2011)), but our modification to accept model-groups is.

${ }^{19} \mathrm{~A}$ quick note: though we borrow the apparatus of research programmes from Lakatos, we do not commit ourselves to the rest of his philosophy.

${ }^{20}$ Progressiveness requires an expansion of the empirical content of a research programme, largely in the form of novel, risky predictions.

${ }^{21}$ We are agnostic on the status of the SM as a model or a theory. Even before the Higgs boson discovery many physicists have called it a theory and that belief has only increased (see Iliopoulos (2014), who suggests referring to it as the "Standard Theory"). Because our reading of Lakatos labels it a research programme either way, the status of the SM is irrelevant to our argument.

${ }^{22}$ As they put it, this "autonomy is the result of two components (1) the fact that models function in a way that is partially independent of theory and (2) in many cases they are constructed with a minimal reliance on
} 
believe that Lakatos' basic concepts can be usefully applied to the model dynamics of particle physics.

Groups of models that share common characteristics, like supersymmetry or CH models, are treated by particle physicists like Lakatosian research programmes, with hard cores and positive heuristics. ${ }^{23}$ These are our model-groups, first introduced in Sections 2.6 and $3 .^{24}$ Individual members of a model-group, as exemplified within single arXiv entries, behave like Lakatos's conception of a model (representations created using a 'theoretical core' in order to probe a phase space or suggest experimental tests). This reflects the fact that model-groups act as scientific research programmes within particle physics, taking the same role as series of theories within the general Lakatosian account. Individual instances from a group act as part of the protective belt around its hard core, which is composed of unifying ideas or mathematical frameworks that remain consistent across all its members. ${ }^{25}$

Our reading of Lakatos, in which model-groups can take the place of a series of theories, forms what we call the "model dynamics" of particle physics: the way model-groups grow more complicated, interact with each other, rise in prominence, or drop out of favour. These dynamics are demonstrated in a coarse-grained way in the graphs in Section 3, with model-groups reacting to data collection at the LHC, but also reflecting internal theoretical developments (i.e. the emergence of new tools and techniques) and responding to the occasional puzzle arising from interesting data signatures.

The upshot of our approach to understanding the model landscape is that it provides us with a versatile and nuanced framework for the survival of these model-groups as the Higgs search revealed no BSM indications: the model-groups are research programmes, with changes occurring in their protective belts as LHC data further constrains the phase space of EWSB. These continuously updating protective belts allow physicists to incorporate, adjust for, or otherwise explain data that conflict with individual members while maintaining the hard cores without change. ${ }^{26}$ In her presentation at the EW session of Moriond 2012, Sanz sums up particle physicists' activities in almost Lakatosian terms: "[SUSY and CH models] need gymnastics, model-building, tuning...creativity, in a word, to pass the stringent electroweak precision tests (and the always tightening collider bounds)" (Sanz, 2012).

Supersymmetry forms the largest and best known research programme within particle physics, aside from the SM itself. SUSY's hard core includes an extension of the SM introducing an additional symmetry between fermions and bosons. Its protective belt consists of the various model-group members describing the breaking of this symmetry, at what energy this breaking occurs, and where the proposed superpartners can be found. While some SUSY models remain compatible with the LHC data, others, like cMSSM, are largely excluded (see Bechtle et al. (2016)). Our choice of using $\mathrm{CH}$ models as our case study was motivated by our desire to provide a less familiar example of a particle physics research programme. The other modelgroups are variegated in their approaches to EWSB, and so have equally diverse hard cores. For our comparative data, we've tentatively taken EFT and OPE approaches to constitute a

high level theory" (Morgan and Morrison, 1999, 43).

${ }^{23}$ Indeed, Lakatos uses examples, like the Bohr atom, which seem more like models than theories. Meanwhile, the work being done in, say, SUSY has many of the characteristics Lakatos ascribes to theories.

${ }^{24}$ An earlier classification of models around core ideas appears in ?, where she utilizes the notion of "theoretical cores" from Morrison (2007). However, we feel that the framework of research programmes provides a clearer picture for understanding the model landscape of particle physics.

${ }^{25}$ The boundaries of a model-group can be somewhat porous, as physicists and ideas often move from one to another. Elements are cross pollinated between research programmes. This, however, does not contradict the Lakatosian framework we are using, since it allows for such exchanges as long as each research programme as a whole remains intact.

${ }^{26}$ Our account is not meant to explain why physicists choose to work within a particular research programme. As discussed in the literature (see e.g. ?), the reasons include epistemic and pragmatic virtues. 
model-group, whether or not they represent real models or are merely a set of epistemic search practices. $^{27}$

Before continuing, we will briefly address a potential objection to our use of Lakatosian research programmes, namely: why are we using research programmes rather than Laudan's research traditions ${ }^{28}$ or some variation of Structural Realism to explain the continuity of models' central components? Research traditions are too broad in their scope, necessitating further arguments to show that the entirety of particle physics does not count as a single tradition. Research programmes, however, already appropriately describe the observed macro-level behaviour. Concerning Structural Realism, it isn't clear that it is mathematical structures that are being preserved, as the case of EWSB shows, there is a liberal importing of structures from one domain into another without any realist aspirations. The case of Technicolor (TC) models described below further demonstrates how core ideas can be passed down, without keeping relevant mathematical structures. Hard cores are broad enough to capture the essence of what survives over time beyond these structures. We will now turn to a case study concerning $\mathrm{CH}$ Higgs models in order to demonstrate in more detail how a model-group behaves in the face of the difficult empirical findings outlined in Section 2.

\subsubsection{Composite Higgs: An Example}

Currently, $\mathrm{CH}$ models are increasingly disfavoured, because the observed properties of the Higgs boson are SM-like, while the free parameters of $\mathrm{CH}$ models have to be stretched to accommodate the data. Yet, papers within the $\mathrm{CH}$ model-group continue to appear on the arXiv in small numbers.

The CH model-group has adjusted to null results as Lakatos described: the hard core of the model-group (that EWSB is attributable to a strong dynamical process caused by new gauge interactions, so that the particle of EWSB, if it exists at all, is composite rather than the fundamental, point-like scalar described in the SM) remains, even as the protective belt shifts in order to account to the less than favourable empirical results. The protective belt itself is made up of various models and strategies describing the phenomenology of these new gauge interactions and the physical phenomena associated with them, in particular, the parameters of the composite particle of EWSB and the new particles introduced by the strong interaction scale $\mathrm{CH}$ models posit. Portions of the protective belt rise and fall in prominence according to the shifting constraints on the parameter space, predominantly caused by the lack of BSM signals.

The idea of a composite system originated at the start of the search for the mass generation of the $\mathrm{W}$ and $\mathrm{Z}$ bosons, piggybacking on the success of superconductivity. Higgs introduced an 'immoderate speculation' (Wells, 2016) when he proposed an elementary scalar boson as a consequence of EWSB. Mass generation by a composite system, where compositeness acts at some scale $f$, attracted a lot of attention, because it circumvents the naturalness problem ${ }^{29}$ and could even offer a DM candidate. This long history, the ability to explain EWSB without a scalar particle, and the fact that it acts as a solution to some problems of the SM has drawn some physicists to the $\mathrm{CH}$ model-group. ${ }^{30}$

\footnotetext{
${ }^{27} \mathrm{We}$ will reserve a more detailed discussion of EFTs and OPEs for another occasion.

${ }^{28}$ See Laudan (1978).

${ }^{29}$ The naturalness problem arises from the significant differences in scale between various SM parameters. Solving it has long driven model builders in particle physics (see e.g. Giudice (2013)).

${ }^{30}$ In defending the motivations to pursue any BSM programme, Peskin claims

In seeking an explanation for electroweak symmetry breaking, we could just write down the minimal Lagrangian available. However, for me, it is much more attractive to look for a theory in which electroweak symmetry breaking emerges from a definite physical idea. If the idea is a profound one, it will naturally lead to new phenomena that we can discover in experiments. (Peskin, 1997, 65)
} 
The first time a composite particle is explicitly mentioned is in (Goldman and Vinciarelli, 1974), though the idea was fleshed out by Susskind (1979); Dimopoulos and Susskind (1979) in the guise of TC, a conceptual descendent of Quantum Chromodynamics. TC is based on a local gauge symmetry representing a new interaction at the $\mathrm{TeV}$ scale. Gauge bosons acquire mass by coupling to Technihadrons, including a scalar that can serve as a Higgs impostor. In these models the vacuum expectation value, $v$, approximates $f$, implying the mass of the Technipion is too large to accommodate a $125 \mathrm{GeV}$ signal. Even before the LHC discovery, however, TC had difficulty accounting for fermion masses without inducing processes that have been experimentally excluded. Several variants were devised, keeping the core TC idea under the names 'extended TC,' 'walking TC,' 'Topcolor-assisted TC,' etc. TC models largely failed to explain the precision data of LEP and the LHC, so have fallen out of favour.

In the 1980s models were suggested based on the breaking of the global symmetry of a new strong interaction. These models could naturally accommodate a light (pseudo) NambuGoldstone boson (pNGB) with $v \ll f$, generating the Higgs potential through radiative corrections. As a consequence, the parameters of these new $\mathrm{CH}$ models are likewise affected by the mass of the Higgs boson they predict, leading to differences in the expected couplings. It took until the late 1990s and early 2000s for viable models to be produced, including the 'Little Higgs' (LH) models. ${ }^{31}$ However, LH models predicted a Higgs mass that was still too high. Once it became clear that the Higgs would be light, LH models became disfavoured. Especially after the December 2011 seminar, more extensive efforts went into explaining a light Higgs mass within the $\mathrm{CH}$ context, ${ }^{32}$ or discussing alternative interpretations of the excess mass around $125 \mathrm{GeV}$. As part of the introduction of pNGBs into CH models, tools like 'partial compositeness' (Kaplan, 1991) were used to allow physicists to solve mass generation in the fermion sector by mixing the SM fermions with new heavy (vector-like) fermions. ${ }^{33}$ Partial compositeness became a potent tool for describing the fermion sector and keeping the Higgs mass low in the $\mathrm{CH}$ model-group. Recall that to be progressive, a research programme must expand its empirical content through risky, testable predictions. We see an example of this forward-looking problem-shift in (Azatov and Galloway, 2012), which analyses the phase space of a light Higgs under the conditions of partial compositeness in a paper first appearing on the arXiv prior to the December 2011 presentation of preliminary results, building on the work of Kaplan (1991) and Giudice et al. (2007).

A search of the arXiv for CH papers citing Kaplan's 1991 paper finds twelve such articles posted prior to 2012, but more than 120 additions since, a ten-fold increase. ${ }^{34}$ Prior to the Higgs boson discovery, there was nothing particularly noteworthy about models built using partial compositeness. After the properties of the Higgs were shown to disfavour or outright exclude many available $\mathrm{CH}$ models, partial compositeness (and Higgs as pNGB generally) was used to explain both the small Higgs mass and the lack of SM deviations found in earlier experiments, increasing the prominence of this conceptual tool on the arXiv.

After the July 2012 observation announcement, a great deal of the $\mathrm{CH}$ parameter space seemed immediately excluded, because the mass was too low for most models developed with the $\mathrm{CH}$ hard core. Determining the particle's couplings became paramount, since deviations from those described in the SM are predicted for all BSM models. One promising finding noted in Section 2.3 was an excess in the relative signal strength in the di-photon channel found by

\footnotetext{
${ }^{31}$ See e.g. (Arkani-Hamed et al., 2002; Agashe et al., 2005).

${ }^{32}$ See e.g., the discussions in (Redi and Tesi, 2012) and (Marzocca et al., 2012).

${ }^{33}$ Fermion mass generation was another problem for TC models.

${ }^{34}$ Using the search terms: "find c Nucl Phys B365 259 and d 1991->2011 and (k "Higgs model: composite" or k "Higgs particle: composite")' and 'find c Nucl Phys B365 259 and d 2012->2017 and (k "Higgs model: composite" or k "Higgs particle: composite")'.
} 
ATLAS and CMS (see Peskin (2012)). These unexpected results provided promising work for theorists working on BSM models, but it was too early in the data analysis process to make any clear determinations, since the errors on many channels were still fairly large. ${ }^{35}$ But the implications of the Higgs announcement for BSM models were quickly apparent: Ellis and You (2012) concluded that many $\mathrm{CH}$ models were excluded and that the newly discovered particle "does indeed walk and quack very much like a Higgs boson".

TC models, to the extent that they still appeared in 2012, also tended to predict high masses for their EWSB particles, if such particles were predicted at all. In the face of the July 2012 discovery, Higgsless models seemed immediately excluded. For instance, the ICHEP presentation of Pomarol (2012) displays an image of a tombstone labelled 'Technicolor Models,' while declaring Higgsless models were dead. However, he also anticipated a short-lived strategy employed by some BSM models: arguing that the newly announced particle was a Higgs impostor. A common variation on this theme was to posit that the $125 \mathrm{GeV}$ particle was the result of the gauge field posited within TC (see e.g. (Eichten et al., 2012)). As more data was generated and analysed, this prospect became less likely. Although TC models still appear on the arXiv, mentions are frequently negative.

In addition to admitting a light Higgs, $\mathrm{CH}$ models describing the Higgs as a pNGB suppressed the expected deviations from the SM that would have been noticed by older experiments. Some of these models make predictions that are potentially testable at the LHC, however. For instance, one of the consequences of a $125 \mathrm{GeV}$ Higgs is fermion resonances in the $\mathrm{TeV}$ scale (see (Marzocca et al., 2012)). In classic Lakatosian fashion, the adjustments to the $\mathrm{CH}$ model-group's protective belt introduced by Higgs-as-pNGB were packaged along with additional tweaks that would need to be addressed, advancing the theoretical scope of the programme. For instance, Carracciolo et al. (2013) discuss additional open problems in accommodating SM fermion masses within partial compositeness, while Panico et al. (2013) make a case for extensive searches at the LHC for top partners described by $\mathrm{CH}$ models.

The CH model-group persists, despite the evidence from the LHC indicating a SM Higgs boson. Until recently, not much has changed as more precision tests have been carried out (leading to the further shrinking of the parameter space for alternatives). As shown in Section 3, entries for $\mathrm{CH}$ models still appear on the arXiv regularly, even experienced a slight increase over their pre-discovery numbers. Many of these entries are meant to seriously advance the programme, rather than criticize it. For example, a paper posted on the arXiv in March of 2016 (Harnik et al., 2017) offers two different partially composite models, along with accompanying phenomenologies. These models contain predictions that may now be testable at the LHC, offering the kind of risky predictions that could expand the empirical content of a Lakatosian research programme. However, there has been a downturn in the prevalence of $\mathrm{CH}$ models (alongside other BSM model-groups) recently. This downturn, if it continues, suggests that the $\mathrm{CH}$ model-group has finally entered a phase where it's protective belt is beginning to fail. If so, then we are approaching the point where we can judge this research programme to be degenerative.

Our case study has been necessarily brief, but it demonstrates the explanatory power of our reading of Lakatos. Only a small sample of arXiv entries was required to trace the strategies utilized by the $\mathrm{CH}$ model-group to account for the Higgs discovery. What this sample shows, however, is that the $\mathrm{CH}$ model-group acts as a Lakatosian research programmes undergoing problem-shifts in the face of empirical challenges. The research programme had to adapt, first to a smaller than expected Higgs mass, then to the firm discovery of a EWSB particle, and then to a lack deviations from SM branching ratios. These adaptations allowed the $\mathrm{CH}$ research

\footnotetext{
${ }^{35}$ There were attempts to explain the strange di-photon channel findings using CH models. Chala (2013), for example, provides a CH model that utilizes the excess which also provides a DM candidate.
} 
programme to persist, even after data was gathered that would have falsified it within other understandings of scientific growth.

\section{Conclusion}

The story of the Higgs confirmation and its theoretical and experimental environment is an interesting case study for the confirmation process and model dynamics. We used a two-pronged approach to investigate the discovery and ensuing confirmation of the Higgs boson, alongside the changing status of alternative theories of EWSB.

Our qualitative approach showed a natural periodization of the discovery process. There were well-defined steps in confirmation that culminated in the acceptance of the boson as the SM Higgs, while at the same time a widely-shared openness remained (and remains) that the particle may not in fact be the Higgs boson of the SM. We have distilled the following points from our above discussion on confirmation: First, a hypothesis can be considered highly confirmed in a certain range, even if there is disconfirming evidence outside of that range. Second, different sets of evidence have different weights; not all measurements are assigned an equal importance. Third, the effect of some particular evidence on the confirmation of competing hypotheses can, in some instances, be in conflict with the confirmation status of the theory as a whole. In order to avoid a conflict, one must recognize that, fourth, a lack of evidence for predictions is relevant for confirmation, but only where there is sufficient experimental sensitivity. The fraction of a theory's parameter space that is not excluded by experiment should not be taken as a matter of confirmation. We take this study to reveal some of the limits of a strictly logical approach to confirmation and identified some of the more important features of confirmation that could bring a logical analysis in line with what we have seen in practice.

In our quantitative approach, we saw that some BSM models offering disfavoured accounts of EWSB are still featured in papers on the arXiv. ${ }^{36}$ We explain this by considering the modelgroups as Lakatosian research programmes, where individual models form a protective belt around the core of a model-group and allow it to survive what might be seen as a disconfirmation. The ideas of strong dynamics in EWSB and extended Higgs sectors remain active in research in part due to their remaining available phase space and their potential to fill gaps in the SM. However, it also is apparent that without the expansion of empirical content, these alternative models appear to be entering a degenerative phase.

Both our approaches taken together have allowed us to dissect the discovery and confirmation process in a way that shows the complexity, and the extended time-line, of the acceptance and rejection of scientific hypotheses.

\section{A Appendix}

\section{A.1 Search Terms}

The following is a list of the search terms that were used in calls to the INSPIRE system for the model-groups.

Composite Higgs Models: find k "technicolor" or k "Higgs model: composite" or k "Higgs particle: composite" or k "Higgs particle: Goldstone particle" or k "pNGB" or k "top: condensation" or k "little Higgs model" or (k "dynamical symmetry breaking" and k "Higgs*") and not (k "supersymmetry*" or k "minimal supersymmetric standard model")

\footnotetext{
${ }^{36}$ Though, we also find that within our time frame their numbers have begun steadily decreasing.
} 
SUSY-Extended Models: find k "minimal supersymmetric standard model" or k "MSSM" or k "supersymm*" and k "Higgs*"

Non-SUSY-Extended Models: find k "2HDM" or "Higgs particle: doublet: 2" or "Higgs particle: triplet" or "Higgs particle: doublet: 3" or "Higgs particle: charged particle" and not (k "supersymmetry*" or k "minimal supersymmetric standard model")

Extra Dimensional Models: find k "Higgsless" or k "Higgsless model" or (k "RandallSundrum model" and k "Higgs*") or (k "dilaton" and k "Higgs*") or (k "radion" and k "Higgs*") or (k "warped" and k "Higgs*") or (k "holography" and k "Higgs*") or (k "higher-dimensional" and k "Higgs*")

Model Independent: find k "effective field theory" or k "operator product expansion" or k "decay: exotic" or k "Higgs particle: invisible decay" and k "Higgs*"

Standard Model Higgs: find (k "experimental results" or k "precision measurement") and k "Higgs particle*" or k "coupling: Higgs" or k "Higgs particle: doublet" or k "Higgs particle: width" or k "Higgs particle: production" and not $\mathrm{k}$ "technicolor*" and not $\mathrm{k}$ "Higgs model: composite" and not k "Higgs particle: composite" and not k "new physics*" and not k "supersymmetry*" and not $\mathrm{k}$ "dark matter*" and not k "Higgs particle: charged particle" and not $\mathrm{k}$ "minimal supersymmetric standard model"

\section{References}

Agashe, K., Contino, R., and Pomarol, A. (2005). The minimal composite Higgs model. Nuclear Physics B, 719:165-187. DOI: 10.1016/j.nuclphysb.2005.04.035. arXiv:hep-ph/0412089.

Arkani-Hamed, N., Cohen, A. G., Katz, E., and Nelson, A. E. (2002). The littlest Higgs. Journal of High Energy Physics, 034. DOI: 10.1088/1126-6708/2002/07/034. arXiv:hep-ph/0206021.

ATLAS Collaboration (2011). $\quad$ ATLAS experiment presents latest
Higgs search status.
atlas-experiment-presents-latest-higgs-search-status. Accessed: 2016-04-11.

ATLAS Collaboration (2012a). Combined search for the standard model Higgs boson using up to $4.9 \mathrm{fb}-1$ of pp collision data at $\operatorname{sqrt}(\mathrm{s})=7 \mathrm{TeV}$ with the ATLAS detector at the LHC. Physics Letters $B, 710(1): 49-66$.

ATLAS Collaboration (2012b). Observation of a new particle in the search for the standard model Higgs boson with the ATLAS detector at the LHC. Physics Letters B, 716:1-29. arXiv:1207.7214 [hep-ex].

Azatov, A. and Galloway, J. (2012). Light custodians and Higgs physics in composite models. Physical Review D, 85:055013. DOI: 10.1103/PhysRevD.85.055013. arXiv:1110.5646v2 [hepph].

Bechtle, P. et al. (2016). Killing the cMSSM softly. Eur. Phys. J., C76(2):96.

Bhattacharya, S. and Jain, S. (2016). A review of the discovery of SM-like Higgs boson in $\mathrm{H} \rightarrow \gamma \gamma$ decay channel with the CMS detector at the LHC. Pramana, 87(3):35. 
Campbell, R. and Vinci, T. (1983). Novel confirmation. Philosophy of Science, 34(4).

Camporesi, T. (2012). Workshop summary and perspectives. Presented at the Higgs Coupling 2012 workshop, Tokyo, Japan, November 18-20, 2012.

Carracciolo, F., Parolini, A., and Serone, M. (2013). UV completions of composite Higgs models with partial compositeness. Journal of High Energy Physics, 066. DOI: 10.1007/JHEP02(2013)066. arXiv:1211.7290 [hep-ph].

Chala, M. (2013). $h \rightarrow \gamma \gamma$ excess and dark matter from composite Higgs models. Journal of High Energy Physics, 122. DOI: 10.1007/JHEP01(2013)122. arXiv:1210.6208 [hep-ph].

CMS Collaboration (2011). CMS search for the standard model Higgs boson in LHC data from 2010 and 2011. http://cms.web.cern.ch/news/ cms-search-standard-model-higgs-boson-lhc-data-2010-and-2011. Accessed: 201604-11.

CMS Collaboration (2012a). Combined results of searches for the standard model Higgs boson in pp collisions at sqrt(s) $=7 \mathrm{TeV}$. Physics Letters B, 710(1):26-48.

CMS Collaboration (2012b). Observation of a new boson at a mass of $125 \mathrm{GeV}$ with the CMS experiment at the LHC. Physics Letters B, 716:30-61. arXiv:1207.7235 [hep-ex].

Dawid, R. (2017). Bayesian perspectives on the discovery of the Higgs particle. Synthese, 194:377-394.

Denisov, D. (2013). Moriond QCD 2013 experimental summary. Presented at the XLVIIIth Rencontres de Moriond for QCD and High Energy Interactions, La Thuile, Italy, March 9-16, 2013. arXiv:1306.6908 [hep-ex].

Dimopoulos, S. and Susskind, L. (1979). Mass without scalars. Nuclear Physics B, 155:237-252.

Dissertori, G. (2012). Moriond 2012, QCD and high energy interactions: Experimental summary. Presented at the XLVIIth Rencontres de Moriond for QCD and High Energy Interactions, La Thuile, Italy, March 10-17, 2012. arXiv:1205.2209 [hep-ex].

Dittmaier, S., Mariotti, C., Passarino, G., and Tanaka, R. (2011). Handbook of LHC Higgs cross sections: 1. inclusive observables. arXiv:1101.0593v3 [hep-ph].

Eichten, E., Lane, K., and Martin, A. (2012). A Higgs imposter in low-scale technicolor. arXiv:1210.5462 [hep-ph].

Ellis, J. and You, T. (2012). Global analysis of the Higgs candidate with mass $125 \mathrm{GeV}$. Journal of High Energy Physics, JHEP09(2012):123. DOI: 10.1007/JHEP09(2012)123. arXiv:1207.1693 [hep-ph].

Englert, F. and Brout, R. (1964). Broken symmetry and the mass of gauge vector mesons. Physical Review Letters, 13(9):321-323.

Franklin, A. (2013). Shifting Standards: Experiments in Particle Physics in the Twentieth Century. University of Pittsburgh Press, Pittsburgh, PA.

Friederich, S., Harlander, R., and Karaca, K. (2014). Philosophical perspectives on ad hoc hypotheses and the Higgs mechanism. Synthese, 191(16):3897-3917. 
Giudice, G. F. (2013). Naturalness after LHC8. Presented at High Energy Physics Conference of the European Physical Society [EPS HEP], Stockholm, Sweden, July 18-24, 2013. arXiv:1307.7879 [hep-ph].

Giudice, G. F., Grojean, C., Pomarol, A., and Rattazzi, R. (2007). The strongly-interacting light Higgs. Journal of High Energy Physics, JHEP06(2007):045.

Goldman, T. and Vinciarelli, P. (1974). Composite Higg field and finite symmetry breaking in gauge theories. Physical Review D, 10:3431-3434.

Grojean, C. (2012). Theoretical implications of the Higgs discovery. Presented at the Higgs Coupling 2012 workshop, Tokyo, Japan, November 18-20, 2012.

Guralnik, G., Hagen, C. R., and Kibble, T. W. (1964). Global conservation laws and massless particles. Physical Review Letters, 13(20):585-587.

Haber, H. E., Hempfling, R., and Hoang, A. H. (1997). Approximating the radiatively corrected Higgs mass in the minimal supersymmetric model. Zeitschrift für Physik C Particles and Fields, 75(3):539-554.

Harnik, R., Howe, K., and Kearney, J. (2017). Tadpole-induced electroweak symmetry breaking and pNGB Higgs models. Journal of High Energy Physics, 111. doi: 10.1007/JHEP03(2017)111. arXiv:1603.03772 [hep-ph].

Hempel, C. G. (1965). Studies in the Logic of Confirmation, pages 3-46. Free Press, New York.

Higgs, P. (1964a). Broken symmetries and the masses of gauge bosons. Physical Review Letters, 13(16):508-509.

Higgs, P. (1964b). Broken symmetries, massless particles and gauge fields. Physical Review Letters, 12(2):132-133.

Howson, C. and Urbach, P. (1991). Bayesian reasoning in science. Nature, 350(6317):371-374.

Iliopoulos, J. (2014). Theory summary talk. Presented at the XLVIXth Rencontres de Moriond for Electroweak Interactions and Unified Theories, La Thuile, Italy, March 15-22, 2014.

Jeffrey, R. C. (1992). Probability and the art of judgement. Cambridge studies in probability, induction, and decision theory. Cambridge University Press, Cambridge.

Johansson, L.-G. and Matsubara, K. (2011). String theory and general methodology: A mutual evaluation. Studies in History and Philosophy of Science Part B: Studies in History and Philosophy of Modern Physics, 42:199-210.

Kaplan, D. B. (1991). Flavor at SSC energies: A new mechanism for dynamically generated fermion masses. Nuclear Physics B, 365:259-278.

Kraml, S. (2013). Implications of the $125 \mathrm{GeV}$ Higgs for Supersymmetry. In Helmholtz Alliance Linear Collider Forum: Proceedings of the Workshops Hamburg, Munich, Hamburg 2010-2012, Germany, pages 366-375, Hamburg. DESY, DESY.

Lakatos, I. (1978). Falsification and the methodology of scientific research programmes. In Currie, G. and Worrall, J., editors, The Methodology of Scientific Research Programmes: Philosophical Papers Volume 1. Cambridge University Press, New York. 
Laudan, L. (1978). Progress and Its Problems: Towards a Theory of Scientific Growth. University of California Press.

Laudan, L. and Leplin, J. (1991). Empirical equivalence and underdetermination. Journal of Philosophy, 88(9):449-472.

Mangano, M. L. (2012). Hadron collider physics symposium: The incomplete summary. Presented at the 23rd Hadron Collider Physics Symposium, Kyoto, Japan, November 12-16, 2012.

Mangano, M. L. (2013). Moriond QCD 2013 experimental summary. Presented at the XLVIIIth Rencontres de Moriond for QCD and High Energy Interactions, La Thuile, Italy, March 9-16, 2013. arXiv:1306.6908 [hep-ex].

Marzocca, D., Serone, M., and Shu, J. (2012). General composite Higgs models. Journal of High Energy Physics, JHEP08(2012):013. DOI: 10.1007/JHEP08(2012)013. arXiv:1205.0770 [hep-ph].

Mayo, D. G. (1991). Novel evidence and severe tests. Philosophy of Science, 58(4):523-552.

Mayo, D. G. (2000). Experimental practice and an error statistical account of evidence. Philosophy of Science, $67(3): 207$.

Moretti, L. (2002). For a Bayesian account of indirect confirmation. Dialectica, 56(2):153-173.

Morgan, M. and Morrison, M. (1999). Models as Mediators: Perspectives on Natural and Social Science. Cambridge University Press, Cambridge.

Morrison, M. (2007). Where have all the theories gone. Philosophy of Science, 74:195-228.

O'Luanaigh, C. (14 March 2013). New results indicate that new particle is a Higgs boson. http://home.cern/about/updates/2013/03/ new-results-indicate-new-particle-higgs-boson. Accessed: 2017-04-15.

Panico, G., Redi, M., Tesi, A., and Wulzer, A. (2013). On the tuning and the mass of the composite Higgs. Journal of High Energy Physics, 051. DOI: 10.1007/JHEP03(2013)051. arXiv:1210.7114 [hep-ph].

Peskin, M. (2012). Theoretical summary lecture for Higgs hunting 2012. Presented at the 3rd Higgs Hunting Workshop: Discussions on Tevatron and LHC Results, Orsay, France, July 18-20, 2012. arXiv:1208.5152v2 [hep-ph].

Peskin, M. E. (1997). Beyond the standard model. In Ellis, N. and Neubert, M., editors, High-Energy Physics. Proceedings, pages 49-142. European School, Carry-le-Rouet, France. arXiv:hep-ph/9705479.

Pomarol, A. (2012). Electroweak symmetry breaking - status/directions. Presented at the 36th International Conference on High Energy Physics, Melbourne, Australia, July 4-11, 2012.

Popper, K. R. (1963). Conjectures and Refutations. Routledge and Kegan Paul, London.

Redi, M. and Tesi, A. (2012). Implications of a light Higgs in composite models. Journal of High Energy Physics, JHEP10(2012):166. DOI: 10.1007/JHEP10(2012)166. arXiv:1205.0232 [hep-ph]. 
Sanz, V. (2012). Signatures of non-standard electroweak symmetry breaking. Presented at the XLVIIth Rencontres de Moriond for Electroweak Interactions and Unified Theories, La Thuile, Italy, March 3-10, 2012. arXiv:1207.1912 [hep-ph].

Schurz, G. (2014). Bayesian pseudo-confirmation, use-novelty, and genuine confirmation. Studies in History and Philosophy of Science Part A, 45(1):87-96.

Sphicas, P. (2013). Experimental summary. Presented at the XLVIIIth Rencontres de Moriond for Electroweak Interactions and Unified Theories, La Thuile, Italy, March 2-9, 2013.

Susskind, L. (1979). Dynamics of spontaneous symmetry breaking in the Weinberg-Salam theory. Physical Review D, 20:2619-2625.

't Hooft, G. (1971). Renormalizable Lagrangians for massive Yang-Mills fields. Nuclear Physics, Section B, 35:167-188.

Wells, J. D. (2016). The theoretical physics ecosystem behind the discovery of the Higgs boson. arXiv:1609.04268v1 [physics.hist-ph].

Zwirner, F. (2013). Theory summary. Presented at the XLVIIIth Rencontres de Moriond for Electroweak Interactions and Unified Theories, La Thuile, Italy, March 2-9, 2013. arXiv:1310.3292 [hep-ph]. 